WU-B 93-07

September 1993

\title{
OCTET-BARYON FORM FACTORS IN THE DIQUARK MODEL因
}

\author{
R. Jakob, P. Kroll四, and M. Schürmann \\ Fachbereich Physik, Universität Wuppertal, \\ D-42097 Wuppertal, Germany \\ W. Schweiger \\ Institut für Theoretische Physik, Universität Graz, \\ A-8010 Graz, Austria
}

\begin{abstract}
:
We present an alternative parameterization of the quark-diquark model of baryons which particularly takes care of the most recent proton electric form-factor data from the E136 experiment at SLAC. In addition to electromagnetic form factors of the nucleon, for which good agreement with data is achieved, we discuss the weak axial vector form factor of the nucleon as well as electromagnetic form factors of $\Lambda$ and $\Sigma$ hyperons. Technical advance in calculating the pertinent analytic expressions within perturbative quantum chromodynamics is gained by formulating the wave function of the quark-diquark system in a covariant way. Finally, we also comment on the influence of Sudakov corrections within the scope of the diquark model.
\end{abstract}

PACS: 13.40.Fn; 12.35.Ht; 14.20.Oh

${ }^{*}$ Sponsored in part by the Bundesministerium für Forschung and Technologie, FRG under contract number $06 \mathrm{Wu} 765$

${ }^{1}$ E-mail: kroll@wpts0.physik.uni-wuppertal.de 


\section{INTRODUCTION}

In order to deal with exclusive hadronic reactions at moderately large momentum transfers $\left(Q^{2} \gtrsim 4 \mathrm{GeV}^{2}\right)$ we have advocated a model in which baryons are treated as quark-diquark systems [1]-[4]. This model incorporates scalar (S) and vector (V) diquarks and relies on factorization of short- and long-distance dynamics - i.e., a hadronic amplitude is expressed as a convolution of a hard-scattering amplitude $\widehat{T}$, calculable within perturbative quantum chromodynamics (QCD), with distribution amplitudes (DAs) $\Phi$ which contain the (nonperturbative) binding of the hadronic constituents. Hence, the diquark model can be considered as a modification of the pure quark hard-scattering picture (HSP) [5] which is generally believed to be the correct description of hard exclusive processes at asymptotically large momentum transfers $\left(Q^{2} \rightarrow \infty\right)$. In fact, diquarks are only an effective way to cope with non-perturbative effects still present in the kinematic range we are interested in. One example for this kind of effects is the large asymmetry of the quark momentum distribution inside the nucleon found by means of QCD sum rules [6]. Just such an asymmetric DA occurs to be necessary to explain the nucleon magnetic form-factor data within the pure quark HSP. It is a clear indication for the existence of strong two-quark correlations inside the nucleon [7].

The main ingredients of the diquark model are baryon DAs in terms of quarks and diquarks, the coupling of gluons and photons to diquarks, and, in order to account for the composite nature of diquarks, phenomenological vertex functions (diquark form factors). The proper choice of the diquark form factors guarantees the compatibility of the diquark model with the pure quark HSP in the limit $Q^{2} \rightarrow \infty$. With a common set of parameters specifying the diquarks and process independent DAs for the involved hadrons a good description of electromagnetic nucleon form factors (in the space-like region) [1], electroexcitation of $\Delta$ and $S_{11}$ resonances [3], Compton scattering off protons $(\gamma p \rightarrow \gamma p)$ [2], and photoproduction of Kaons $\left(\gamma p \rightarrow K^{+} \Lambda\right)$ [4], [8] has been accomplished. Thereby, $\mathrm{V}$ diquarks prove to be essential in describing spin effects caused by flips of the baryon helicity. These spin effects are also of non-perturbative origin and play an important role in most exclusive hadronic processes at experimentally accessible values of the momentum transfer $Q^{2}$. They cannot be explained within the pure quark HSP, in which they are suppressed by powers of $(\tilde{m} / Q)(\tilde{m}$ denotes a mass of the order of the hadronic mass).

The parameters of the diquark model were essentially determined by a fit to the world data on elastic electron-nucleon scattering for $Q^{2} \gtrsim 3.5 \mathrm{GeV}^{2}$ (cf. [1]). With the exception of elastic electron-proton differential cross sections [9], which fix the proton magnetic form factor $G_{M}^{p}\left(Q^{2}\right)$ very accurately up to $Q^{2} \approx 31 \mathrm{GeV}^{2}$, the quality and quantity of these data was rather poor. As a consequence there still remained ambiguities in the model parameters, in particular those for the $\mathrm{V}$ diquarks. Very recently, however, better data on the electric form factor of the proton $G_{E}^{p}\left(Q^{2}\right)$ in the momentum-transfer range $1.75 \mathrm{GeV}^{2} \leq Q^{2} \leq 8.83 \mathrm{GeV}^{2}$ have been published [10]. In reproducing the new data our model falls somewhat short. It 
is thus one of the goals of the present paper to reconcile the diquark-model results with experiment. As can be checked most easily in the Breit frame this affects primarily the parameters of the $\mathrm{V}$ diquarks. In that frame the Pauli form factor $F_{2}^{p}\left(Q^{2}\right)$, which determines the difference between $G_{E}^{p}\left(Q^{2}\right)$ and $G_{M}^{p}\left(Q^{2}\right)$, only contributes to helicity-flip transitions of the proton. Such transitions, as we already mentioned, are generated by $\mathrm{V}$ diquarks.

An additional reason that makes a readjustment of the diquark parameters necessary is the introduction of covariant quark-diquark wave functions. According to the HSP, neglecting intrinsic transverse momenta, we treat quarks and diquarks as free on-shell particles with momenta collinear to that of the parent baryon. Therefore the valence (quark-diquark) Fock state of a baryon can be written in a covariant way in terms of the baryonic momentum and helicity (and of course a colour and flavour part). The derivation of such a representation parallels the one for covariant wave functions in the heavy quark effective theory [11. The use of covariant wave functions has many technical advantages. One works with hadronic quantities (spinors, helicities, momenta, ...) from the beginning. The calculation of a large set of scattering amplitudes at the constituent level, which finally have to be combined to a hadronic amplitude, is avoided; one immediately projects onto hadronic states. Last not least, covariant wave functions turn out to be extremely helpful in algebraic computer programs, the use of which is unavoidable for calculating processes more complicated than electron-nucleon scattering. Their introduction, however, slightly changes the original analytic results for the $\mathrm{V}$-diquark contributions. The modifications are related to helicity flips of the quarks neglected in our previous work.

A further objective of this paper is to provide predictions for two types of form factors not considered till now within the diquark model, namely the axial vector form factor of the nucleon and electromagnetic form factors of hyperons. For the first one there exist already a few data up to $Q^{2}=3 \mathrm{GeV}^{2}[12]$. These can be compared with our model results if one assumes that the usual dipole parameterization gives the right trend for higher values of $Q^{2}$. On the other hand, for hyperon form factors no data are available at present. Nevertheless, their consideration might become of interest if plans will be realized to measure these form factors by scattering a high-quality hyperon beam on an atomic electron target [13]. We obtain results for the hyperon form factors by employing a flavour dependent distribution amplitude for the baryon octet.

Eventually, we also want to comment on the influence of Sudakov suppression in the HSP involving diquarks. Recently Li and Sterman [14, [15] have disputed assertions [16], [17] that perturbative QCD is not applicable at experimentally accessible momentum transfers. The authors of [16] and [17] have pointed out that an asymmetric DA like the one of Ref. [6] strongly enhances contributions from kinematic regions where perturbative QCD does not hold. The main issue of Li's and Sterman's work is that such contributions are suppressed and hence the validity of perturbative QCD is reestablished if one takes into consideration the transverse momenta of the quarks and radiative (Sudakov) corrections. In the light of these findings it is also interesting to know whether a similar mechanism works for diquarks. 
The paper is organized as follows. In Sect. 2 we introduce covariant quark-diquark wave functions and briefly summarize the (Feynman) rules of the diquark model. Sect. 3 contains the results for the electromagnetic form factors of the nucleon with a discussion of Sudakov corrections and the predictions for hyperon form factors. Sect. 4 is devoted to the axial vector form factor of the nucleon. The summary is given in Sect. 5 .

\section{THE HARD-SCATTERING PICTURE WITH DIQUARKS}

As we already mentioned in the introduction there are two elements entering a form factor calculation in the framework of the HSP, namely a hard scattering amplitude $\widehat{T}$ to be calculated in collinear approximation within perturbative QCD and DAs $\Phi$. To leading order in $(1 / Q)$ the form factors are determined by the valence Fock state of the considered hadron - in our model a quark-diquark state in case of an ordinary baryon. For the lowest lying baryon octet, assuming zero relative orbital angular momentum between quark and diquark, the Fock state written in a covariant fashion (omitting the colour part) reads

$$
|B ; p, \lambda\rangle=f_{S} \Phi_{S}^{B}\left(x_{1}\right) \chi_{S}^{B} u(p, \lambda)+f_{V} \Phi_{V}^{B}\left(x_{1}\right) \chi_{V}^{B}\left(\gamma^{\alpha}+p^{\alpha} / m_{B}\right) \gamma_{5} u(p, \lambda) / \sqrt{3}
$$

$u$ is the spinor of the baryon, $p$ and $m_{B}$ its momentum and mass, respectively. The two terms in (2.1) represent configurations consisting of a quark and either an $\mathrm{S}$ or a $\mathrm{V}$ diquark. The Lorentz-index $\alpha$ labels the components of the $\mathrm{V}$-diquark polarization vector. In the region where only a few $\mathrm{GeV}$ of momentum are transferred the mass of the baryon is not completely negligible. If it is taken into account and the sum of the constituents' fourmomenta is demanded to give the baryon four-momentum one ends up with running masses

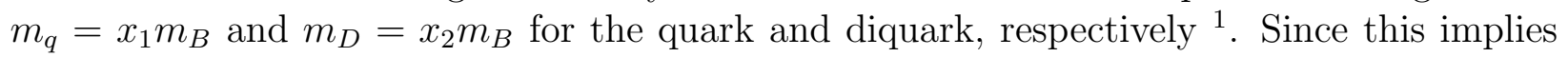
that quark, diquark, and baryon have the same (four) velocity, the chain of arguments leading to covariant wave functions in the heavy quark effective theory [11] can immediately be transferred to our collinear situation to prove (2.1).

We assume an SU(6)-like spin-flavour dependence for the octet-baryon wave functions. Hence the flavour functions $\chi$ for proton, neutron and hyperons take on the form

$$
\begin{gathered}
\chi_{S}^{p}=u S_{[u, d]}, \chi_{V}^{p}=\left[u V_{\{u, d\}}-\sqrt{2} d V_{\{u, u\}}\right] / \sqrt{3}, \\
\chi_{S}^{n}=d S_{[u, d]}, \chi_{V}^{n}=-\left[d V_{\{u, d\}}-\sqrt{2} u V_{\{d, d\}}\right] / \sqrt{3}, \\
\chi_{S}^{\Sigma^{+}}=-u S_{[u, s]}, \chi_{V}^{\Sigma^{+}}=\left[u V_{\{u, s\}}-\sqrt{2} s V_{\{u, u\}}\right] / \sqrt{3}, \\
\chi_{S}^{\Sigma^{-}}=d S_{[d, s]}, \chi_{V}^{\Sigma^{-}}=-\left[d V_{\{d, s\}}-\sqrt{2} s V_{\{d, d\}}\right] / \sqrt{3},
\end{gathered}
$$

\footnotetext{
${ }^{1} x_{1}$ denotes the fraction of the baryon momentum carried by the quark $\left(x_{2}=1-x_{1}\right.$ that of the diquark).
} 


$$
\begin{gathered}
\chi_{S}^{\Sigma^{0}}=\left[d S_{[u, s]}+u S_{[d, s]}\right] / \sqrt{2}, \chi_{V}^{\Sigma^{0}}=\left[2 s V_{\{u, d\}}-d V_{\{u, s\}}-u V_{\{d, s\}}\right] / \sqrt{6}, \\
\chi_{S}^{\Lambda^{0}}=\left[u S_{[d, s]}-d S_{[u, s]}-2 s S_{[u, d]}\right] / \sqrt{6}, \chi_{V}^{\Lambda^{0}}=\left[u V_{\{d, s\}}-d V_{\{u, s\}}\right] / \sqrt{2} .
\end{gathered}
$$

The DA $\Phi_{S(V)}^{B}\left(x_{1}\right)$ is nothing else but a light-cone wave function integrated over transverse momentum. The $r=0$ value of the corresponding configuration space wave function is given by the constant $f_{S(V)}$. For our purposes the phenomenological ansatz

$$
\phi_{S}^{B}\left(x_{1}\right)=N_{S} x_{1} x_{2}^{3} \exp \left[-b^{2}\left(m_{q}^{2} / x_{1}+m_{S}^{2} / x_{2}\right)\right]
$$

for the DA of the S diquark and the slightly more complicated expression

$$
\phi_{V}^{B}\left(x_{1}\right)=N_{V} x_{1} x_{2}^{3}\left(1+c_{1} x+c_{2} x^{2}\right) \exp \left[-b^{2}\left(m_{q}^{2} / x_{1}+m_{V}^{2} / x_{2}\right)\right]
$$

for the DA of the $\mathrm{V}$ diquark occur to be quite appropriate. The analytic form of these DAs originates from a nonrelativistic harmonic-oscillator wave function [18]. Therefore the masses appearing in the exponentials have to be considered as constituent masses. We take $330 \mathrm{MeV}$ for light quarks, $580 \mathrm{MeV}$ for (light) diquarks, and add $150 \mathrm{MeV}$ for each strange quark. The oscillator parameter $b^{2}$ is taken to be $0.248 \mathrm{GeV}^{-2}$, so that the full wave function gives rise to a reasonable value of $600 \mathrm{MeV}$ for the mean intrinsic transverse momentum of quarks inside a nucleon. The "normalization" constants $N_{S}$ and $N_{V}$ are determined by the condition $\int d x_{1} \Phi_{S(V)}\left(x_{1}\right)=1$.

The Feynman diagrams contributing to the hard-scattering amplitude of baryon form factors are displayed in Fig. 1. The gluon- and photon-diquark vertices are defined by

$$
\begin{aligned}
S\left(\begin{array}{l}
g \\
\gamma
\end{array}\right) S: & i\left(\begin{array}{c}
+g_{s} t^{a} \\
-e_{0} e_{S}
\end{array}\right)\left(p_{1}+p_{2}\right)_{\mu}, \\
V\left(\begin{array}{c}
g \\
\gamma
\end{array}\right) V: & i\left(\begin{array}{c}
-g_{s} t^{a} \\
+e_{0} e_{S}
\end{array}\right)\left[g_{\alpha \beta}\left(p_{1}+p_{2}\right)_{\mu}-\right. \\
& \left.g_{\mu \alpha}\left[\left(1+\kappa_{V}\right) p_{1}-\kappa_{V} p_{2}\right]_{\beta}-g_{\mu \beta}\left[\left(1+\kappa_{V}\right) p_{2}-\kappa_{V} p_{1}\right]_{\alpha}\right],
\end{aligned}
$$

with $g_{s}=\sqrt{4 \pi \alpha_{s}}$ designating the QCD coupling constant, $\kappa_{V}$ the anomalous (chromo)magnetic moment of the vector diquark and $t^{a}\left(=\lambda^{a} / 2\right)$ the Gell-Mann colour matrix 日. Gauge invariance also calls for contact terms

$$
\begin{aligned}
\gamma S g S & :-2 i e_{0} e_{S} g_{s} t^{a} g_{\mu \nu} \\
\gamma V g V & : \quad+i e_{0} e_{V} g_{s} t^{a}\left(2 g_{\mu \nu} g_{\alpha \beta}-g_{\mu \beta} g_{\alpha \nu}-g_{\mu \alpha} g_{\beta \nu}\right) .
\end{aligned}
$$

\footnotetext{
${ }^{2}$ The different signs in electromagnetic $\left(e_{0}>0\right)$ and strong vertices are due to the fact that the diquark is in a colour state belonging to the antitriplet.
} 
In applications of the diquark model Feynman diagrams are calculated with these rules for point-like particles. In order to take into account the composite nature of diquarks phenomenological vertex functions (diquark form factors) have to be introduced. Our choice

$$
\begin{aligned}
& F_{S}^{(3)}\left(Q^{2}\right)=\delta_{S} \frac{Q_{S}^{2}}{Q_{S}^{2}+Q^{2}}, \\
& F_{V}^{(3)}\left(Q^{2}\right)=\delta_{V}\left(\frac{Q_{V}^{2}}{Q_{V}^{2}+Q^{2}}\right)^{2},
\end{aligned}
$$

for 3-point functions and

$$
F_{S}^{(n)}=a_{s} F_{S}^{(3)}\left(Q^{2}\right), \quad F_{V}^{(n)}=a_{V} F_{V}^{(3)}\left(Q^{2}\right)\left(\frac{Q_{V}^{2}}{Q_{V}^{2}+Q^{2}}\right)^{(n-3)}
$$

for n-point functions $(n \geq 4)$ ensures that in the limit $Q^{2} \rightarrow \infty$ the diquark model evolves into the pure quark HSP. The factor $\delta_{S(V)}=\alpha_{s}\left(Q^{2}\right) / \alpha_{s}\left(Q_{S(V)}^{2}\right)\left(\delta_{S(V)}=1\right.$ for $\left.Q^{2} \leq Q_{S(V)}^{2}\right)$ provides the correct powers of $\alpha_{s}\left(Q^{2}\right)$ for asymptotically large $Q^{2} \cdot \alpha_{S}=12 \pi / 25 \ln \left(Q^{2} / \Lambda_{Q C D}^{2}\right)$ is used with $\Lambda_{Q C D}=200 \mathrm{MeV}$ and restricted to be smaller than 0.5. $a_{S}$ and $a_{V}$ are strength parameters which allow for the possibility of diquark excitation and break-up in intermediate states where diquarks can be far off-shell.

\section{ELECTROMAGNETIC FORM FACTORS}

For baryons belonging to the lowest lying baryon octet $\left(J^{P}=1 / 2^{+}\right)$the matrix elements of the electromagnetic current operator can be cast into the form

$$
\left\langle B ; p_{f}, \lambda_{f}\left|\widehat{J}^{\mu}\right| B ; p_{i}, \lambda_{i}\right\rangle=-i e_{0} \bar{u}\left(p_{f}, \lambda_{f}\right)\left[\gamma^{\mu} G_{M}^{B}\left(Q^{2}\right)-\frac{\kappa_{B}}{2 m_{B}}\left(p_{f}+p_{i}\right)^{\mu} F_{2}^{B}\left(Q^{2}\right)\right] u\left(p_{i}, \lambda_{i}\right),
$$

with $\kappa_{B}$ denoting the anomalous magnetic moment of the baryon $B$. The magnetic form factor $G_{M}^{B}$ and the Pauli form factor $F_{2}^{B}$ are phenomenological functions of $Q^{2}=\left(p_{f}-p_{i}\right)^{2}$ which parameterize the electromagnetic structure of the baryon $B$. The two other commonly used form factors are the electric form factor $G_{E}^{B}$ and the Dirac form factor $F_{1}^{B}$. They are related to $G_{M}^{B}$ and $F_{2}^{B}$ via $F_{1}^{B}=G_{M}^{B}-\kappa_{B} F_{2}^{B}$ and $G_{E}^{B}=G_{M}^{B}-\kappa_{B}\left(1+Q^{2} /\left(4 m^{2}\right)\right) F_{2}^{B}$, respectively.

At the constituent level the electromagnetic current is obtained as a convolution of hard scattering amplitudes $\widehat{T}$, given by the Feynman diagrams of Fig.1, with the pertinent DAs (2.8) and (2.9) for $\mathrm{S}$ and $\mathrm{V}$ diquarks, respectively. The covariant formulation (2.1) of the baryon wave functions enters the calculation of the Feynman diagrams as far as the spinor $u\left(p_{q}, \lambda_{q}\right)$ of an external incoming quark has to be replaced by the spinor of the baryon $u(p, \lambda)$ to which it belongs and the polarization vector $\epsilon^{\alpha}\left(p_{V}, \lambda_{V}\right)$ of an external incoming $\mathrm{V}$ diquark 
has to be replaced by the factor $\left(\gamma^{\alpha}+p^{\alpha} / m_{B}\right) \gamma_{5}$ put in front of the baryon spinor $u(p, \lambda)$. For outgoing quarks we have instead $\bar{u}(p, \lambda)$ and for outgoing $\mathrm{V}$ diquarks $\gamma_{5}\left(\gamma^{\alpha}+p^{\alpha} / m_{B}\right)$ at the right hand side of this spinor. Thereby it is already taken into account that the $\lambda= \pm 1 / 2$ helicity state of a baryon consists of a combination of two different quark- $\mathrm{V}$ diquark helicity states 3 . Thus, only baryonic quantities show up in the analytic expressions for the Feynman diagrams. After some commutations and subsequent use of the Dirac equation $\left(\not p u(p, \lambda)=m_{B} u(p, \lambda)\right)$ one immediately ends up with expressions for the various contributions to the electromagnetic current which have the same covariant structure as (3.17). Folding these expressions with the DAs yields

$$
\begin{aligned}
S_{3}\left(Q^{2}\right) & =C_{F} \frac{4 \pi}{Q^{2}} f_{S}^{2} \int_{0}^{1} d x_{1} d y_{1} \Phi_{S}\left(y_{1}\right) 2 \frac{\alpha_{s}\left(\tilde{Q}_{22}^{2}\right)}{x_{2} y_{2}} F_{S}^{(3)}\left(\tilde{Q}_{22}^{2}\right) \Phi_{S}\left(x_{1}\right), \\
S_{4}\left(Q^{2}\right) & =C_{F} \frac{4 \pi}{Q^{2}} f_{S}^{2} \int_{0}^{1} d x_{1} d y_{1} \Phi_{S}\left(y_{1}\right) 2 \frac{\alpha_{s}\left(\tilde{Q}_{11}^{2}\right)}{x_{1} y_{1}} F_{S}^{(4)}\left(\tilde{Q}_{11}^{2}+\tilde{Q}_{22}^{2}\right) \Phi_{S}\left(x_{1}\right), \\
V_{3}\left(Q^{2}\right) & =C_{F} \frac{4 \pi}{9 m_{B}^{2}} f_{V}^{2} \int_{0}^{1} d x_{1} d y_{1} \Phi_{V}\left(y_{1}\right) \frac{\alpha_{s}\left(\tilde{Q}_{22}^{2}\right)}{x_{2} y_{2}} F_{V}^{(3)}\left(\tilde{Q}_{22}^{2}\right) \Phi_{V}\left(x_{1}\right) \kappa_{V}, \\
V_{4}\left(Q^{2}\right) & =C_{F} \frac{4 \pi}{9 m_{V}^{2}} f_{V}^{2} \int_{0}^{1} d x_{1} d y_{1} \Phi_{V}\left(y_{1}\right) \frac{\alpha_{s}\left(\tilde{Q}_{11}^{2}\right)}{x_{1} y_{1}} F_{V}^{(4)}\left(\tilde{Q}_{11}^{2}+\tilde{Q}_{22}^{2}\right) \Phi_{V}\left(x_{1}\right) \\
& \left(\kappa_{V}^{2}\left(1+2 x_{1}+2 y_{1}+x_{1} y_{1}\right)+\frac{1}{2} \kappa_{V}\left(1-\kappa_{V}\right)\left(x_{2}+y_{2}\right)-\frac{3}{2} x_{2} y_{2}\left(1-\kappa_{V}^{2}\right)\right),
\end{aligned}
$$

for the Lorentz-invariant functions in front of $\gamma^{\mu}$ and

$$
\begin{aligned}
V_{3}^{f}\left(Q^{2}\right)=-C_{F} \frac{4 \pi}{9 Q^{2} m_{B}} f_{V}^{2} \int_{0}^{1} d x_{1} d y_{1} \Phi_{V}\left(y_{1}\right) \frac{\alpha_{s}\left(\tilde{Q}_{22}^{2}\right)}{x_{2} y_{2}} F_{V}^{(3)}\left(\tilde{Q}_{22}^{2}\right) \Phi_{V}\left(x_{1}\right) \\
\left(4 \kappa_{V}-\left(x_{2}+y_{2}\right)\left(2+3 \kappa_{V}\right)\right), \\
V_{4}^{f}\left(Q^{2}\right)=-C_{F} \frac{4 \pi}{9 m_{V}^{2} m_{B}} f_{V}^{2} \int_{0}^{1} d x_{1} d y_{1} \Phi_{V}\left(y_{1}\right) \frac{\alpha_{s}\left(\tilde{Q}_{11}^{2}\right)}{x_{1} y_{1}} F_{V}^{(4)}\left(\tilde{Q}_{11}^{2}+\tilde{Q}_{22}^{2}\right) \Phi_{V}\left(x_{1}\right) \\
\left(\left(\kappa_{V}+\frac{1}{2}\right)\left(x_{1}+y_{1}\right)+\kappa_{V}-1\right),
\end{aligned}
$$

for the Lorentz-invariant functions in front of $\left(p_{f}+p_{i}\right)^{\mu} . C_{F}=4 / 3$ denotes the colour factor and $\tilde{Q}_{i j}^{2}=x_{i} x_{j} Q^{2} \cdot m_{V}=580 \mathrm{MeV}$ is the mass of the $\mathrm{V}$ diquark. It appears in the $\mathrm{V}$-diquark propagator of the 4-point diagrams. Finally, these contributions have to be multiplied with appropriate charge and flavour factors and summed up in order to obtain $G_{M}^{B}$ and $F_{2}^{B}$.

\footnotetext{
${ }^{3}$ As a consequence of the collinear approximation $p_{q}=x_{1} p$ and $p_{V}=x_{2} p$ this combination $\propto$ $\left(u\left(p_{q}, \lambda\right) \epsilon^{\alpha}\left(p_{V}, 0\right)-\sqrt{2} u\left(p_{q},-\lambda\right) \epsilon^{\alpha}\left(p_{V}, 2 \lambda\right)\right)$ can be rewritten as $\sqrt{x_{1}}\left(\gamma^{\alpha}+p^{\alpha} / m_{B}\right) \gamma_{5} u(p, \lambda)$.
} 


\subsection{Nucleon form factors}

For nucleon form factors the pertinent combinations of scalar and vector n-point contributions read:

$$
\begin{aligned}
G_{M}^{p} & =e_{u} S_{3}+e_{u d} S_{4}+\left(e_{u}+2 e_{d}\right) V_{3}+\left(e_{u d}+2 e_{u u}\right) V_{4}, \\
G_{M}^{n} & =e_{d} S_{3}+e_{u d} S_{4}+\left(e_{d}+2 e_{u}\right) V_{3}+\left(e_{u d}+2 e_{d d}\right) V_{4}, \\
F_{2}^{p} & =-\frac{2 m_{p}}{\kappa_{p}}\left(\left(e_{u}+2 e_{d}\right) V_{3}^{f}+\left(e_{u d}+2 e_{u u}\right) V_{4}^{f}\right) \\
F_{2}^{n} & =-\frac{2 m_{n}}{\kappa_{n}}\left(\left(e_{d}+2 e_{u}\right) V_{3}^{f}+\left(e_{u d}+2 e_{d d}\right) V_{4}^{f}\right) .
\end{aligned}
$$

$e_{q}$ and $e_{q_{1} q_{2}}$ are the electric charges of the quarks and diquarks (consisting of quarks $q_{1}$ and $q_{2}$ ) in units of $e_{0}$. Employing these analytic expressions the parameters of the model are determined by means of

i) the electron-proton elastic differential cross sections measured by Sill et al. [19 which overcome the preliminary results of [9] and are available for $2.9 \leq Q^{2} \leq 31.2 \mathrm{GeV}^{2}$,

ii) the electric and magnetic form factors of the proton for $2.5 \leq Q^{2} \leq 8.83 \mathrm{GeV}^{2}$ obtained by Bosted et al. [10 by means of a Rosenbluth separation,

iii) the ratio $\sigma_{n} / \sigma_{p}$ of the $e n$ and $e p$ differential cross sections extracted from the reaction $e d \rightarrow$ epn at the quasi-elastic peak [20] in the $Q^{2}$ range from $4 \mathrm{GeV}^{2}$ to $10 \mathrm{GeV}^{2}$,

iv) and both the neutron form factors at $Q^{2}=4 \mathrm{GeV}^{2}$ extracted from quasi-elastic $e-d$ cross sections via Rosenbluth separation [21.

We want to emphasize that we only use data which are free from assumptions on relationships between $G_{E}$ and $G_{M}$. A good fit to these data is achieved with the following set of parameters:

$$
\begin{aligned}
f_{S}=73.85 \mathrm{MeV}, Q_{S}^{2} & =3.22 \mathrm{GeV}^{2}, a_{S}=0.15 \\
f_{V}=127.7 \mathrm{MeV}, Q_{V}^{2} & =1.50 \mathrm{GeV}^{2}, a_{V}=0.05, \quad \kappa_{V}=1.39, \quad c_{1}=5.8, c_{2}=-12.5
\end{aligned}
$$

The results for proton and neutron electric and magnetic form factors are displayed in Figs. 2 - 5. For comparison also shown are the predictions of two frequently cited models, the vector meson dominance model of Körner and Kuroda [22], and a hybrid model [23] which combines the features of vector meson dominance with QCD asymptotics. For general properties of the diquark model in connection with nucleon form factors we refer to Ref. [1]. 
Our results for the magnetic and electric form factor of the proton match the data quite well 4. The obvious difference between $G_{M}^{p}$ and $G_{E}^{p}$ signals that $F_{2}^{p}$ is by no means small. This is an indication for sizable helicity flip transitions of the proton.

For the neutron the situation seems not to be that satisfactory. However, we get the correct sign and order of magnitude of the neutron magnetic form factor, anyhow a severe challenge for any model based on perturbative QCD, and also our electric form factor predictions exhibit the right trend at low $Q^{2}$. Missing quantitative agreement with experiment should not be overvalued. Due to the small number of neutron data and their relatively large error bars they are of minor importance for the $\chi^{2}$-value of our fit. With the $Q^{2}$ values of the few data points available in the $\mathrm{GeV}$ region we reach the limit of validity of the diquark model. On the other hand, the extraction of these data from quasi-elastic $e-d$ cross sections is not completely straightforward and cannot be performed model independent. Effects of final-state interactions and meson exchange currents, not taken into account as yet, may still alter the results. Therefore we did not attempt to achieve a better reproduction of the neutron data by giving them an artificially high weight in the parameter-fitting procedure.

In Fig. 6 the four n-point contributions to the magnetic form factors $S_{3}, S_{4}, V_{3}$, and $V_{4}$ are displayed to give some insight in their relative magnitudes. In order to see how they built up the magnetic form factors of the nucleon they have to be combined according to Eqs.(3.24) and (3.25).

At the end of this subsection a few words about the self-consistency of form factor calculations within perturbative QCD are in order. As can be seen from Eqs. (3.18) - (3.23) the running QCD coupling constant $\alpha_{S}$ diverges in the end-point regions $x_{1}, y_{1} \rightarrow 0,1$. The same happens in the pure quark HSP. As a consequence, perturbation theory looses its self-consistency as a weak-coupling expansion. One way out - actually the one we employ - is to "freeze "the running coupling beyond an infrared cutoff [24]. In addition we make use of distribution amplitudes which strongly suppress these dangerous kinematic regions. The infrared cutoff represents a new scale within such an approach. The introduction of this new scale can be avoided if the transverse momenta of the hadronic constituents as well as radiative (Sudakov) corrections are taken into account. Radiative corrections select components of the wave function with small spatial extend. The numerical effect is similar to that of the infrared cutoff. This has been demonstrated recently by Li and Sterman within the pure quark HSP for the form factors of the pion 14 and and the nucleon 15 . They claim that in this way the self-consistency of the perturbative treatment can be reestablished for $Q$ larger than 20 to $30 \Lambda_{Q C D}$ without introducing a scale in addition to $\Lambda_{Q C D}$. We have performed an analogous calculation for the S-diquark contribution to the proton form factor and arrive at the same conclusion. A treatment of the endpoint regions in the manner proposed by Li and Sterman diminishes the results presented above only by 10 to $20 \%$. Since

\footnotetext{
${ }^{4}$ The $G_{M}^{p}$ data of Ref. [19] have been extracted from elastic $e-p$ cross sections under the assumption $G_{M}^{p}=\left(1+\mu_{p}\right) G_{E}^{p}$ which is not satisfied in our model. The agreement with these data is only an indication that the $e-p$ cross section is insignificantly affected by $G_{E}^{p}$.
} 
Sudakov corrections mainly depend on colour and not on spin we suppose a similar behaviour for $\mathrm{V}$ diquarks. That small suppression can be compensated by adjusting the parameters of the model appropriately. However this has not been done in a systematic way. We also note that there is further suppression due to the dependence of the baryon wave function on the intrinsic transverse momentum [25]. We leave the systematic study of this effect and other higher twist contributions to a forthcoming paper.

\subsection{Hyperon form factors}

Employing the SU(6)-type flavour functions (2.4) - (2.7) we are immediately in the position to extend the electromagnetic form-factor calculation to other octet baryons. If one assumes the same DAs (2.8) and (2.9) and the same constants $f_{S}$ and $f_{V}$ for all members of the baryon octet no additional ingredients are needed. A particular SU(6)-breaking scheme is already implied by the different values of $f_{S}$ and $f_{V}$, the different functional form of $\Phi_{S}$ and $\Phi_{V}$, and the mass dependence of the DAs (2.8), (2.9). A similar kind of SU(6) breaking proved also to be appropriate for the treatment of proton-antiproton annihilation into hyperon antihyperon pairs within (a simplified version of) the diquark model [26]. Although no data on electromagnetic hyperon form factors are available at present, predictions for them might become of interest in the future. The experiment proposed in 13 could provide data with reasonable accuracy up to momentum transfers $Q^{2} \approx 2 \mathrm{GeV}^{2}$. Although this $Q^{2}$ value is certainly not large enough for a direct comparison with our predictions, the large- $Q^{2}$ trends provide perhaps some insight.

In terms of the four n-point functions already introduced in (3.18) - (3.21) the magnetic form factors for $\Lambda$ and $\Sigma$ hyperons read:

$$
\begin{aligned}
G_{M}^{\Sigma^{+}}= & e_{u} S_{3}+e_{u s} S_{4}+\left(e_{u}+2 e_{s}\right) V_{3}+\left(e_{u s}+2 e_{u u}\right) V_{4}, \\
G_{M}^{\Sigma^{-}}= & G_{M}^{\Sigma^{+}}(u \leftrightarrow d) \\
2 G_{M}^{\Sigma^{0}}= & \left(e_{u}+e_{d}\right) S_{3}+\left(e_{u s}+e_{d s}\right) S_{4} \\
& +\left(e_{u}+e_{d}+4 e_{s}\right) V_{3}+\left(e_{u s}+e_{d s}+4 e_{u d}\right) V_{4}, \\
2 G_{M}^{\Lambda^{0}}= & \frac{1}{3}\left(e_{u}+e_{d}+4 e_{s}\right) S_{3}+\frac{1}{3}\left(e_{u s}+e_{d s}+4 e_{u d}\right) S_{4} \\
& +3\left(e_{u}+e_{d}\right) V_{3}+3\left(e_{u s}+e_{d s}\right) V_{4} .
\end{aligned}
$$

Similar expressions can be derived for $F_{2}$. From the charge factors in front of the n-point functions it should be obvious whether the single quark is strange or non-strange and correspondingly $150 \mathrm{MeV}$ have to be added to the quark or diquark mass occurring in the exponential of the DAs (2.8), (2.9). Ignoring this mass dependence one can look at Fig.6 to get a first estimate for the hyperon form factors. One finds that

$$
G_{M}^{\Sigma^{+}} \approx G_{M}^{p} \text { and } 2 G_{M}^{\Sigma^{0}} \approx-2 G_{M}^{\Lambda^{0}} \approx-G_{M}^{n}
$$


The magnetic form factors of $\Sigma^{0}$ and $\Lambda^{0}$ are small due to almost perfect cancellation between the dominant contributions $S_{3}$ and $S_{4}$. The full calculation, with the pertinent constituent masses in the DAs (2.8), (2.9) taken into account, confirms these observations as can be seen from Fig.7. For comparison also shown is the result obtained by Chernyak et al. [27] within the pure quark HSP (valid at high $Q^{2}$ ). Within the theoretical uncertainties agreement between the diquark model and the HSP can be observed. At the lower $Q^{2}$-end there exist in addition predictions from a relativized constituent-quark model for $\Lambda^{0}$ and $\Sigma^{0}$ form factors [28]. Also these results lie within the trend of the diquark model. At the end of this section we want to mention that in the $\mathrm{SU}(6)$-limit the relations (3.33) hold exactly. Therefore, the magnetic hyperon form factors could also give us valuable hints at the amount of SU(6) breaking, in particular in the DAs of the hyperons.

\section{THE AXIAL-VECTOR FORM FACTOR OF THE NUCLEON}

Within the HSP the axial-vector form factor of the nucleon $g_{A}$ defined by

$$
\left\langle p ; p_{f}, \lambda_{f}\left|\widehat{A}_{\mu}{ }_{\mu}\right| n ; p_{i}, \lambda_{i}\right\rangle=i \cos \theta_{c} \bar{u}\left(p_{f}, \lambda_{f}\right)\left[\gamma_{\mu} \gamma_{5} g_{A}\left(Q^{2}\right)+\left(p_{f}-p_{i}\right)_{\mu} \gamma_{5} f_{A}\left(Q^{2}\right)\right] u\left(p_{i}, \lambda_{i}\right)
$$

is calculated along the same lines as the electromagnetic form factors. The same set of Feynman diagrams has to be evaluated with the only difference that the photon is replaced by a $W$-boson. No additional parameters enter the calculation of $g_{A}$. Thus, it is an important touchstone for the whole picture and in particular for the DA. Unfortunately data on $g_{A}$ are very scarce above $1 \mathrm{GeV}^{2}$. The only information comes from a measurement of the reaction $\nu_{\mu} n \rightarrow \mu^{-} p$ in a deuterium target [12]. The analysis of the cross section is based on the standard V-A theory. As a consequence of the PCAC hypothesis the contribution from $f_{A}$ becomes negligible. Therefore the (unpolarized) differential cross section suffices to determine $g_{A}$. Analogous to the electromagnetic form factors and in accordance with dimensional counting $g_{A}$ is usually parameterized as

$$
g_{A}\left(Q^{2}\right)=\frac{g_{A}(0)}{\left(1+Q^{2} / M_{A}^{2}\right)^{2}} .
$$

From $\beta$-decay it is known that $g_{A}(0)=1.23 \pm 0.01$. The experiment of Kitagaki et al. [12], with a maximum $Q^{2}$-value of $3 \mathrm{GeV}^{2}$, gives $M_{A}=1.05_{-0.16}^{+0.12} \mathrm{GeV}$. The average over all $\nu$ data yields $M_{A}=1.03 \pm 0.036 \mathrm{GeV}$. But this result is dominated by low $Q^{2}$ data. Be that as it may, $Q^{2}$ is a bit small for application of perturbative QCD. In view of the results for the magnetic form factor of the proton one may, however, hope that the dipole parameterization (4.35) gives the right trend up to, say, 10 or $15 \mathrm{GeV}^{2}$. If this is the case $Q^{4} g_{A}$ is about $1.5_{-0.9}^{+0.7} \mathrm{GeV}^{4}(1.38 \pm 0.20$ from the neutrino data) in that region. 
This is not a severe constraint and causes no difficulty for the pure quark HSP. With the DAs proposed in refs. [6] and [29] Carlson et al. [30] found $Q^{4} g_{A}=1.36$ and $1.00 \mathrm{GeV}^{4}$, respectively. Very recently also Stefanis and Bergmann [31] found a similar result $\left(Q^{4} g_{A}=\right.$ $1.44 \mathrm{GeV}^{4}$ at $Q^{2} \approx 10 \mathrm{GeV}^{2}$ and for $\Lambda_{Q C D}=180 \mathrm{MeV}$ ) with a nucleon DA that combines features of the Chernyak-Zhitnitsky DA [6] and the Gari-Stefanis DA [29].

Within the diquark model the calculation of $g_{A}$ is not as straightforward as in the pure quark HSP. In the 4-point contributions the $\mathrm{W}$ interacts with diquarks and has to change their flavour and in some cases even their spin (e.g.: $W^{+} S_{[u, d]} \rightarrow V_{\{u, u\}}$ ). Taking into account such transitions would require the introduction of new diquark form factors. One may, however, argue that the 4-point contributions are negligible since diquarks break up when they interact with a $\mathrm{W}$. This would mainly lead to particle production. We assume, therefore, the dominance of the 3-point contribution and obtain the simple result

$$
g_{A}=S_{3}-V_{3} .
$$

From Fig.6 it is clear that our prediction for $g_{A}$ agrees reasonably well with the parameterization (4.35). For $Q^{2}=15 \mathrm{GeV}^{2}$ we get $Q^{4} g_{A}=1.15 \mathrm{GeV}^{4}$.

\section{SUMMARY}

In this paper we have presented a new parameterization of the quark-diquark model of baryons. This model was introduced originally in Refs. [1] - [4] in order to describe exclusive hadronic reactions at intermediate momentum transfers. In [1] the parameters of the model were determined by means of elastic electron-nucleon scattering data. A refit of the parameters became necessary due to the publication of new and more accurate electron-nucleon data [10], [19], [20], [21]. With the new set of parameters good agreement with the electric and magnetic form-factor data of the proton for $Q^{2} \gtrsim 4 \mathrm{GeV}^{2}$ has been achieved. We have not attempted to optimize our results concerning the neutron form factors, since corresponding data are still very sparse and only known for $Q^{2} \leq 4 \mathrm{GeV}^{2}$ - the lower limit of validity of the diquark model. Nevertheless, the diquark-model results for the electromagnetic neutron form factors lie within the high $Q^{2}$ trend indicated by the existing experiments. More and better data for the neutron form factors, say between 4 and $20 \mathrm{GeV}^{2}$, would certainly be very helpful and could furthermore facilitate the decision between the different theoretical models applicable in that kinematic region.

In the spirit of Li and Sterman [14], [15] we have also investigated the influence of transverse momentum and Sudakov corrections for the S-diquark part of our model. It turned out that the suppression of the dangerous end-point regions $x \rightarrow 0,1$ by means of such corrections is already implemented in our model in an effective way through the restriction $\alpha_{s} \leq 0.5$ on the strong coupling constant and the particular choice of the DAs. 
Since Sudakov corrections mainly depend on colour and not on spin we suppose them also to be of minor importance for $\mathrm{V}$-diquarks.

In addition to electromagnetic nucleon form factors, we have considered the axial-vector form factor of the nucleon and electromagnetic form factors of $\Lambda$ and $\Sigma$ hyperons. Under the assumption that contributions in which the $\mathrm{W}$ boson couples to the diquarks are negligible - due the necessary change of flavour the diquarks tend to break up - the diquark-model predictions for $g_{A}$ compare reasonably well with the dipole parameterization of $g_{A}$ extracted from $\nu_{\mu} n \rightarrow \mu^{-} p$ data [12]. For the magnetic $\Lambda$ and $\Sigma^{0}$ form factors the diquark model interpolates between the corresponding results of the pure quark HSP [27], valid at high $Q^{2}$ and predictions of a constituent quark model [28], valid at small $Q^{2}$. In the limit of exact $S U(6)$ spin-flavour symmetry the hyperon form factors are related by $G_{M}^{\Sigma^{+}}=G_{M}^{p}$ and $2 G_{M}^{\Sigma^{0}}=-2 G_{M}^{\Lambda^{0}}=-G_{M}^{n}$. Since we have used flavour dependent DAs our results deviate from these relations. Data for these hyperon form factors could give us valuable clues at the amount of $S U(6)$ breaking and the flavour dependence of octet-baryon DAs.

Finally it should be mentioned that there exist already other successful applications of the new diquark-model parameterization presented here. These include the time-like form factor of the proton, $\gamma \gamma \rightarrow p \bar{p}, \eta_{c} \rightarrow p \bar{p}$ [32], and (virtual) Compton scattering off protons 33. 


\section{References}

[1] Kroll, P., Schürmann, M., Schweiger, W.: Z. Phys. A - Hadrons and Nuclei 338, 339 (1991)

[2] Kroll, P., Schürmann, M., Schweiger, W.: Int. J. Mod. Phys. A6, 4107 (1991)

[3] Kroll, P., Schürmann, M., Schweiger, W.: Z. Phys. A - Hadrons and Nuclei 342, 429 (1992)

[4] Kroll, P., Schürmann, M., Schweiger, W.: In: Quark Cluster Dynamics. Goeke, K., Kroll, P., Petry, H.R. (eds.), p.179. Lecture Notes in Physics, Vol.417, Berlin, Heidelberg: Springer-Verlag (1993)

[5] Lepage, G.P., Brodsky, S.J.: Phys. Rev. D22, 2157 (1980)

[6] Chernyak, V.L., Zhitnitsky, I.R.: Nucl. Phys. B246, 52 (1984)

[7] Stefanis, N.G.: Phys. Rev. D40, 2305 (1989)

[8] Schürmann, M.: Internal report WUB-DIS 92-4, Wuppertal (1992)

[9] Arnold, R.G., et al.: Phys. Rev. Lett. 57, 174 (1986)

[10] Bosted, P.E., et al.: Phys. Rev. Lett. 68, 3841 (1992)

[11] Georgi, H.: Nucl. Phys. B348, 293 (1991)

[12] Kitagaki, T., et al.: Phys. Rev. D28, 436 (1983)

[13] Garkusha, V.I., et al.: preprint IHEP 90-81, Protvino (1990)

[14] Li, H., Sterman, G.: Nucl. Phys. B381, 129 (1992)

[15] Li, H.: preprint ITP-SB-92-25, Stony Brook (1992);

[16] Isgur, N., Llewellyn Smith, C.H.: Nucl. Phys. B317, 526 (1989)

[17] Radyushkin, A.V.: Nucl. Phys. A532, 141c (1991)

[18] Huang, T.: Nucl. Phys. (Proc. Suppl.) B7, 320 (1989)

[19] Sill, A.F., et al.: Phys. Rev. D48, 29 (1993)

[20] Rock, S., et al.: Phys. Rev. D46, 24 (1992)

[21] Lung, A., et al.: Phys. Rev. Lett. 70, 718 (1993)

[22] Körner, J.G., Kuroda, M.: Phys. Rev. D16, 2165 (1977) 
[23] Gari, M., Krümpelmann, W.: Z. Phys. A - Atoms and Nuclei 322, 689 (1985)

[24] Cornwall, J.M.: Phys. Rev. D26, 1453 (1982)

[25] Jakob, R., Kroll, P.: preprint CERN-TH.6900/93 (1993), to be published in Phys. Lett. B

[26] Kroll, P., Quadder, B., Schweiger, W.: Nucl. Phys. B316, 373 (1989)

[27] Chernyak, V.L., Ogloblin, A.A., Zhitnitsky, I.R.: Z. Phys. A - Particles and Fields 42, 569 (1989)

[28] Warns, M., Pfeil, W., Rollnik, H.: Phys. Lett. B258, 431 (1991)

[29] Gari, M., Stefanis, N.G.: Phys. Rev. D35, 1074 (1987)

[30] Carlson, C.E., Gari, M., Stefanis, N.G.: Phys. Rev. Lett. 58, 1308 (1987)

[31] Stefanis, N.G., Bergmann, M.: Phys. Rev. D47, R3685 (1993)

[32] Kroll, P., Pilsner, Th., Schürmann, M., Schweiger, W.: preprint CERN-TH.6869/93 (1993), to be published in Phys. Lett. B

[33] Kroll, P., Guichon, A.M., Schürmann, M.: preprint WUB-93-31, Wuppertal (1993)

[34] Höhler, G., et al.: Nucl. Phys. B114, 505 (1976)

[35] Walker, R.C., et al.: Phys. Lett. B224, 353 (1989)

[36] Akerlof, C.W., et al.: Phys. Rev. 135, B810 (1964)

[37] Hanson, K.M., et al.: Phys. Rev. D8, 753 (1973)

[38] Bartel, W., et al.: Nucl. Phys. B58, 429 (1973) 


\section{FIGURE CAPTIONS}

Fig.1 Diagrams contributing to electromagnetic and weak form factors of baryons. The double lines represent either an $\mathrm{S}$ or a $\mathrm{V}$ diquark.

Fig.2 The magnetic form factor of the proton. Shown are the results of the diquark model (solid line), the vector-meson-dominance model [22] (dotted line), and a hybrid model

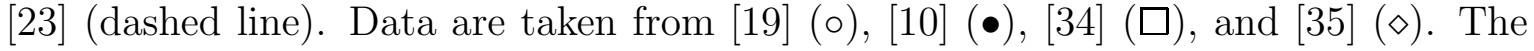
lower solid line shows the Pauli form factor $F_{2}^{p}$ as resulting from the diquark model.

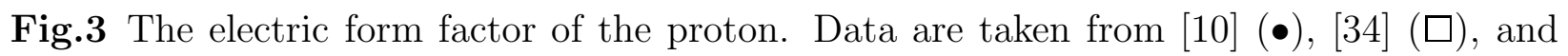
[35] ( $\diamond)$. Curves same as in Fig.2.

Fig.4 The magnetic form factor of the neutron. Data are taken from [36] $(\triangle),[37$ ] $\diamond)$, 338 $(\bullet)$, and 21] (०). Curves same as in Fig.2.

Fig.5 The electric form factor of the neutron. Data are taken from [36] $(\triangle)$, [37] $(\diamond)$, [38] $(\bullet)$, and 21] (०). Curves same as in Fig.2.

Fig.6 The 3- and 4-point terms contributing to the magnetic form factors of the nucleon (cf. (3.24) and (3.25)).

Fig.7 Our predictions for the magnetic form factors of $\Lambda$ and $\Sigma$ hyperons. The dashes and squares represent the corresponding results from the pure quark HSP [27] and a relativized constituent quark model [28], respectively. 
This figure "fig1-1.png" is available in "png" format from: http://arxiv.org/ps/hep-ph/9310227v1 

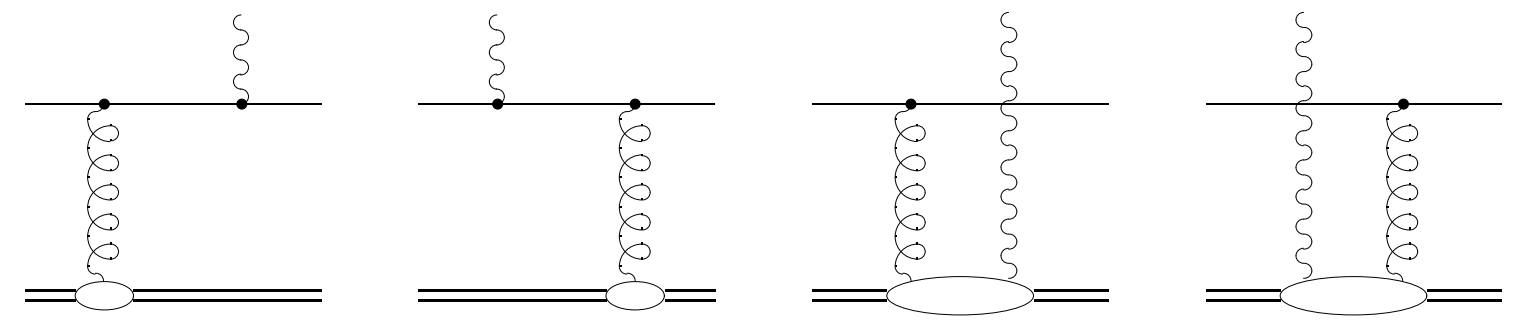

Fig.1 
This figure "fig1-2.png" is available in "png" format from: http://arxiv.org/ps/hep-ph/9310227v1 


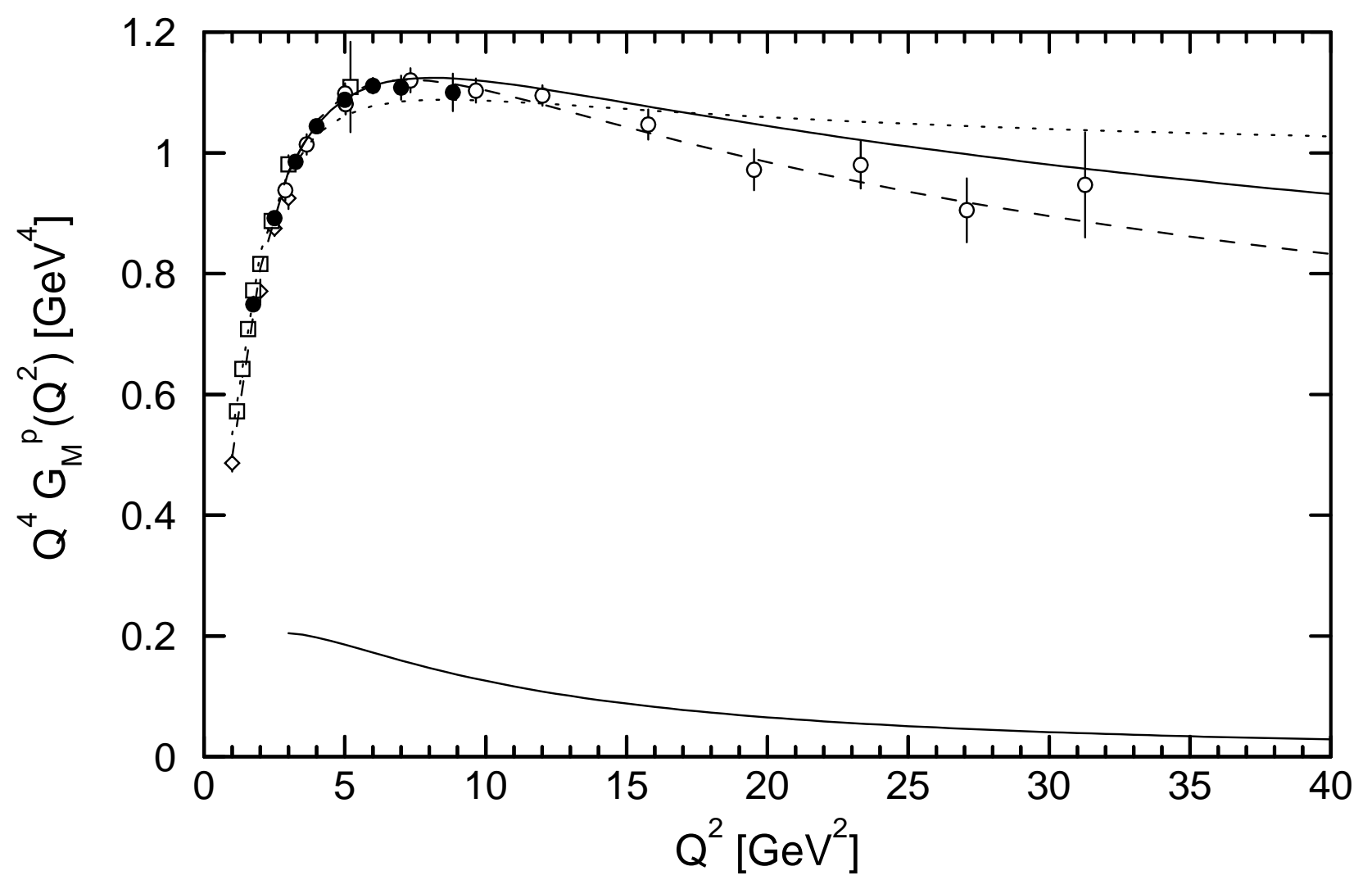

Fig.2 
This figure "fig1-3.png" is available in "png" format from: http://arxiv.org/ps/hep-ph/9310227v1 


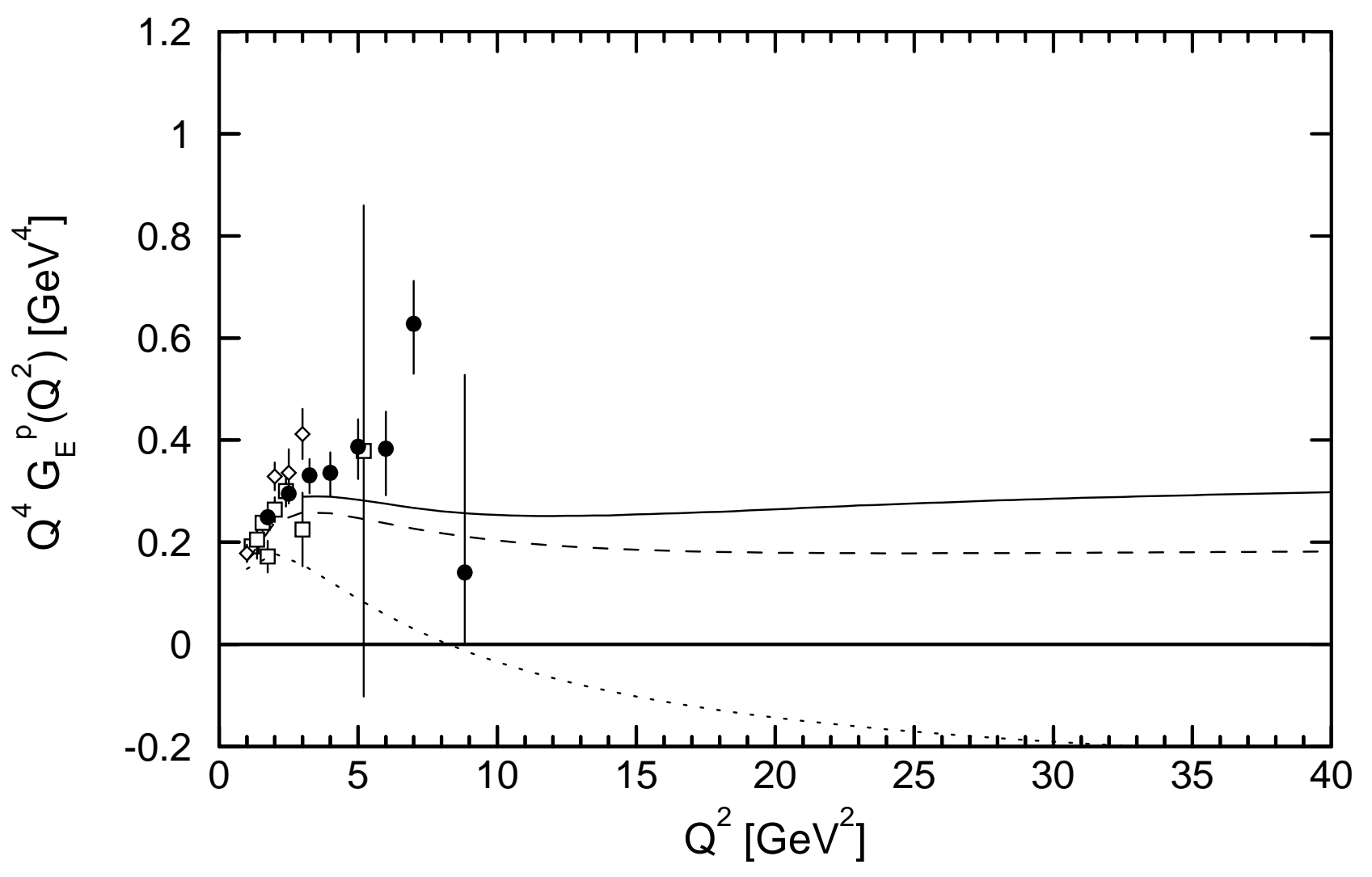

Fig.3 
This figure "fig1-4.png" is available in "png" format from: http://arxiv.org/ps/hep-ph/9310227v1 


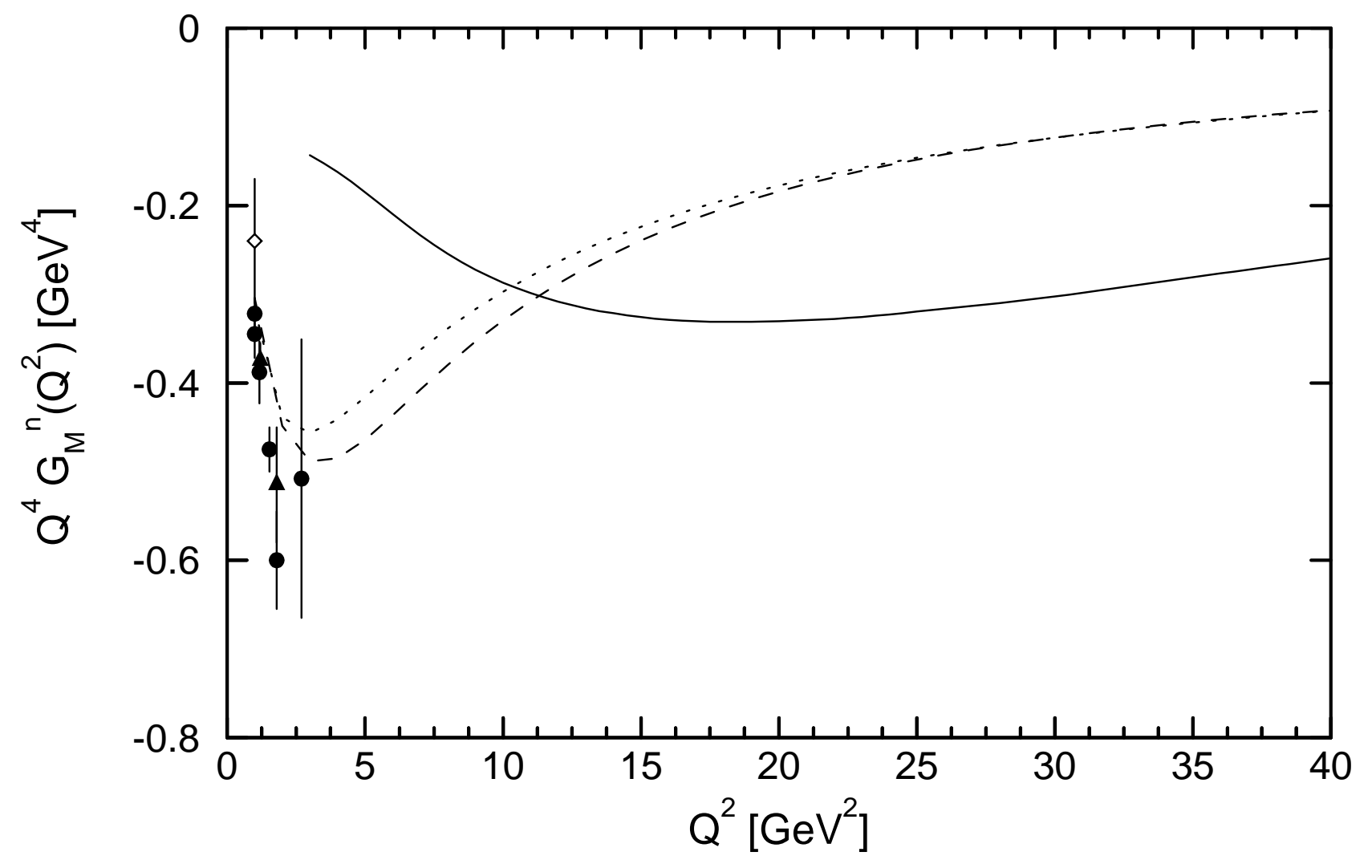

Fig.4 


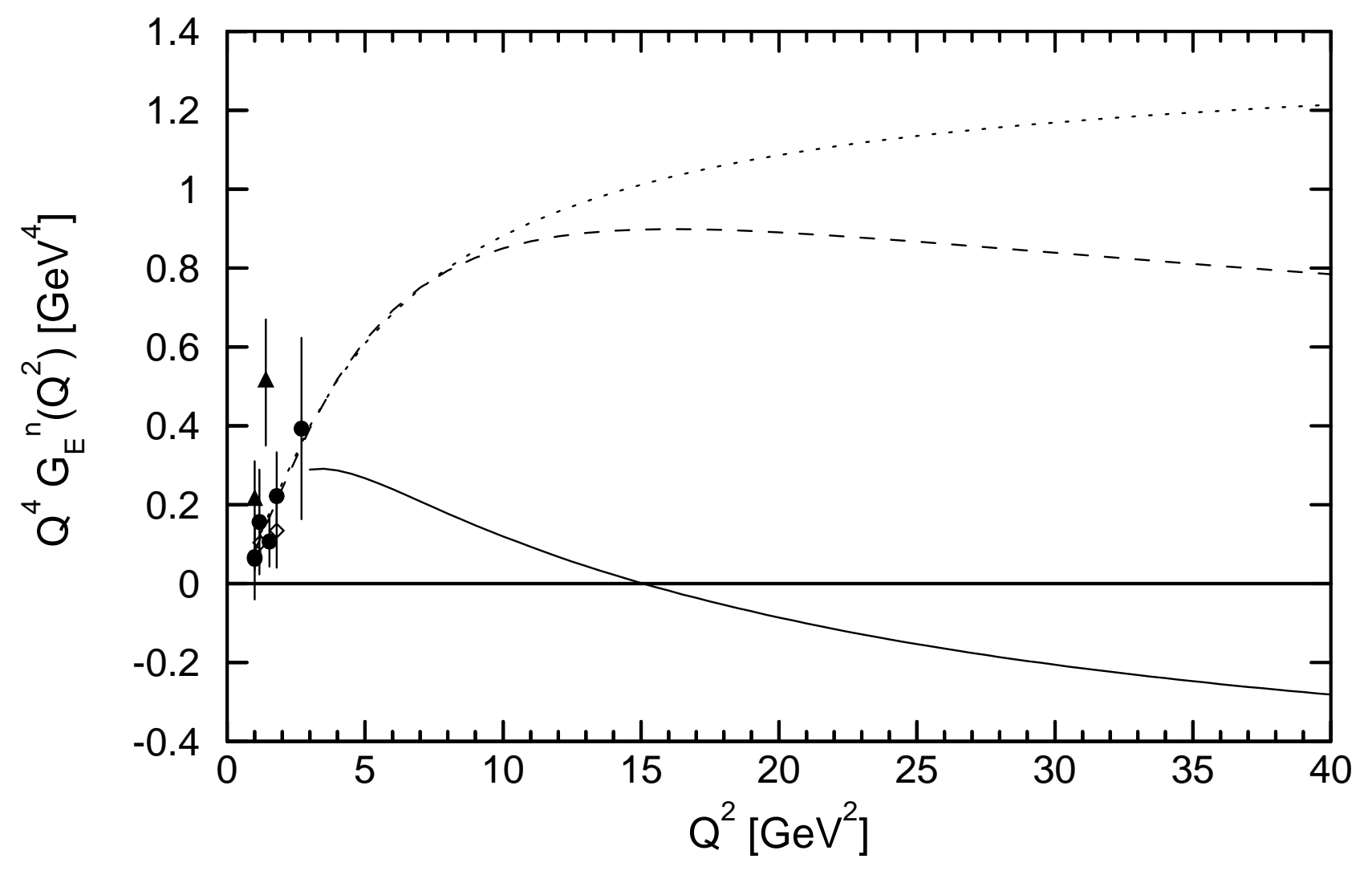

Fig.5 


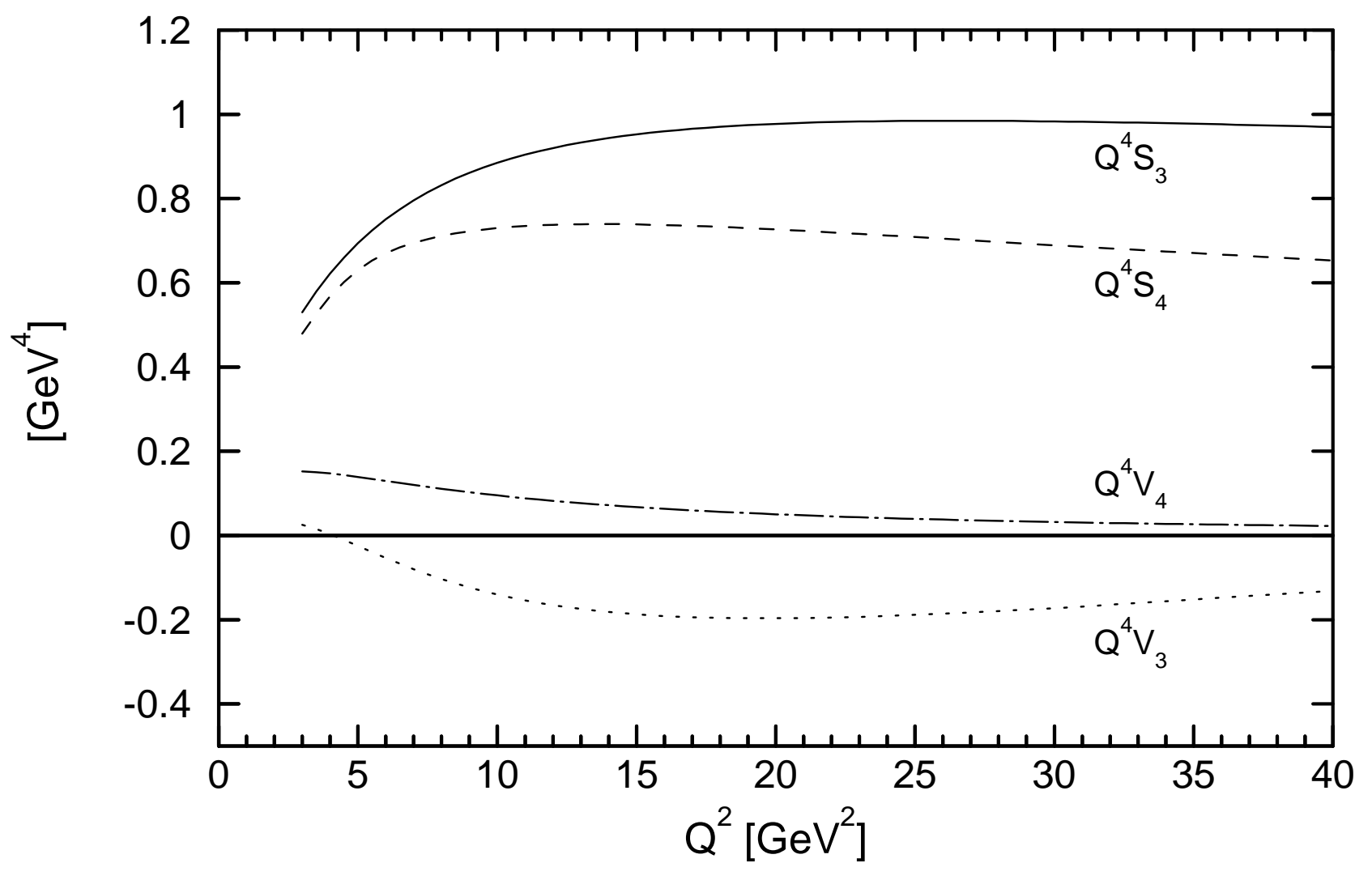

Fig.6 


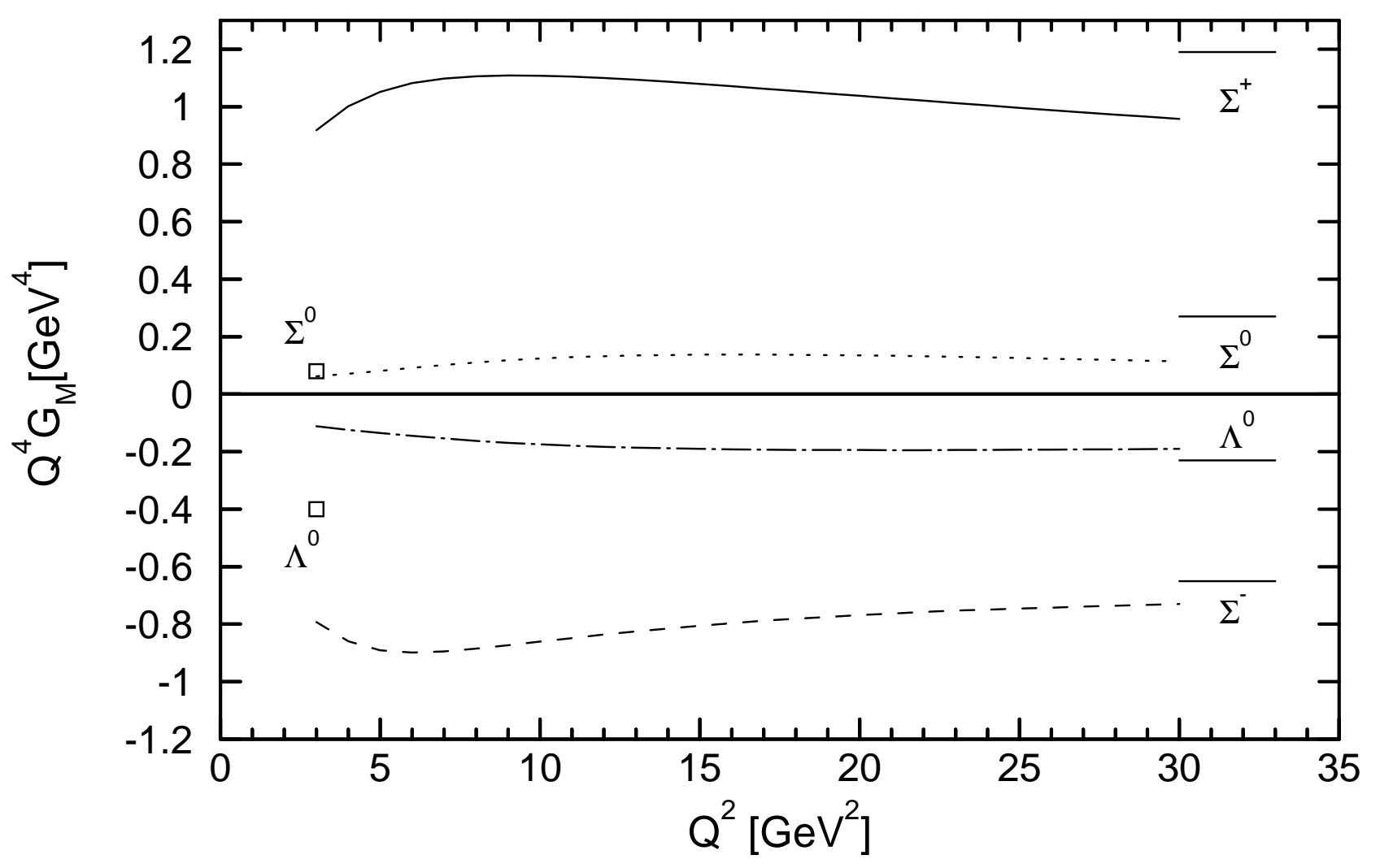

Fig.7 